\title{
Observation of the a-C:H run-in behaviour for dry forming applications of aluminium
}

\author{
Tim Abraham ${ }^{1, *}$, Günter Bräuer ${ }^{1}$, Felix $\mathrm{Kretz}^{2}$, and Peter Groche ${ }^{2}$ \\ ${ }^{1}$ Fraunhofer-Institute for Surface Engineering and Thin Films, 38108 Braunschweig, Germany \\ ${ }^{2}$ Institute for Production Engineering and Forming Machines, Darmstadt University of Technology, 64287 Darmstadt, Germany
}

\begin{abstract}
Amorphous hydrogenated carbon coatings (a-C:H) are well known for their exceptional tribological properties and are established as tool coatings for numerous forming applications. However, utilized in dry forming processes of aluminium a premature failure of an a-C:H coated tool often occurs due to strong adhesive wear. In this paper the run-in behaviour of a-C:H is investigated and as a possible reason for the premature tool failure evaluated. Therefore, oscillating ball-on-disc tribometer tests and strip drawing tests, for a more realistic emulation of real forming processes, will be conducted. According to these tests, the run-in period of a-C:H coatings is characterized by a high friction value and adhesion tendency and thus is decisive for the tool performance. Based on a subsequent analysis of the coating wear, the predominating wear mechanisms during the run-in period are discussed. The intrinsic nanomater-scale a-C:H roughness is identified as a crucial factor determining the tribological properties of the run-in behaviour. By reducing the coating roughness prior to the forming process, the adhesion tendency and friction value can be reduced significantly. The results demonstrate the tribological performance of pre-treated a-C:H coatings for dry sheet metal forming of aluminium EN AW-5083.
\end{abstract}

Keyword: Metal forming, Coating, Tribology

\section{Introduction}

Due to its weight advantage and excellent energy absorption capacity, aluminium is an excellent material for a wide range of applications, for example in the automotive or aerospace industry. However, the design of forming technologies for aluminium alloys presents some challenges. Especially in dry forming processes, the strong adhesion tendency of aluminium alloys leads to high adhesive wear on forming tools [1] and affects the surface quality of components, the process stability and the targeted tolerances. As a consequence of the adhesive wear, the production of aluminium alloy components is currently limited to lubricated forming processes. In order to develop effective strategies to reduce adhesive wear and enable dry sheet metal forming processes, the relevant wear mechanisms and influencing parameters must be considered.

One solution to optimize the tribological properties of forming tools and to enable dry sheet metal forming of aluminium alloys is the deposition of hard thin film coatings. Amorphous hydrogenated carbon coatings (a$\mathrm{C}: \mathrm{H})$ are well known for their exceptional tribological properties [2]. Combining a low friction value and a high wear resistance, a-C:H coatings are already in use for several forming applications. Numerous investigations reported a low adhesion tendency in contact with aluminium $[3,4,5]$. The low adhesion tendency of a-C:H coatings was attributed to the intrinsic capability of selflubrication and high inertness of the passivated surface $[6,7]$. Nevertheless, forming tools coated with state of the art a-C:H coatings still fail due to a rapid formation of aluminium adhesions comparable with uncoated forming tools.

A commonly known characteristic of hard thin film coatings is the run-in behaviour. In general, this behaviour describes a short period at the beginning of a sliding contact with differing tribological properties. Luo et. al. [8] and Goldsmith et. al. [9] reported an increased friction value and wear during the run-in period of a-C:H sliding against sapphire $(\mu \sim 0.20)$ and aluminium oxide $(\mu \sim$ 0.63 ) without lubrication. This differing tribological behaviour could explain the rapid formation of aluminium adhesions in dry forming processes of aluminium and may be the reason for a premature tool failure.

In this paper an a-C:H coating system is systematically investigated to define the tribological properties and wear mechanisms during the run-in behavior. Furthermore, the impact of these results on dry forming processes of aluminium is evaluated in strip drawing tests, which enable a more realistic emulation of real forming processes.

\section{Experimental setup}

\subsection{Coating preparation and characterisation}

The a-C:H coating system was deposited by a plasma assisted chemical vapour deposition process (PACVD) with acetylene $(\mathrm{C} 2 \mathrm{H} 2)$ as the precursors gas combined with argon as process gas. To enhance the coating adhesion, the substrates were sputtered clean for $30 \mathrm{~min}$ at the beginning of the coating process and an interlayer

* Corresponding author: tim.abraham@ist.fraunhofer.de 
based on titanium with a thickness of $0.2 \mu \mathrm{m}$ was applied by magnetron sputtering. The thickness of the complete a$\mathrm{C}: \mathrm{H}$ coating system measured $2.2 \mu \mathrm{m}$. A more detailed description of the deposition process and the coating characteristics is stated in [10].

Additional to the substrates for the tribological tests, polished flat samples made of bearing steel (1.3505) were coated to allow a subsequent analysis of the coating properties. Coating hardness HUpl was determined with a commercial instrument (Fischerscope H 100) recording load versus depth curves up to $30 \mathrm{mN}$ measuring 34.1 GPa. The abrasive wear rate of $1.010^{-15} \mathrm{~m}^{3} / \mathrm{Nm}$ was determined with the ball cratering test [11] operating with an alumina (A12O3) suspension (mean alumina grain size $1 \mu \mathrm{m})$.

\subsection{Tribological tests}

The run-in behaviour of the a-C:H coating was investigated with an oscillating ball-on-disc tribometer test using a Universal Mechanical Tester (UMT-3, Bruker Corporation). Therefore, the a-C:H coating was deposited on steel balls and tested in a dry sliding contact against aluminium sheets. The coated steel balls measured 10 $\mathrm{mm}$ in diameter and were made of bearing steel (1.3505). The aluminium sheets were made of EN AW-5083 in a mill finished $\mathrm{H} 111$ condition with a thickness of $1.5 \mathrm{~mm}$. Roughness data of the test substrates were collected using a profilometer (Taylor-Hubson) and are summarized in Table 1. The ball-on-disc tests were conducted three times to improve the statistical quality of the friction and wear values. The amplitude of the oscillation measured $20 \mathrm{~mm}$.

Table 1. Roughness of the test substrates.

\begin{tabular}{|l|l|l|}
\hline & $\begin{array}{l}\mathbf{R a} \\
{[\boldsymbol{\mu} \mathbf{m}]}\end{array}$ & $\begin{array}{l}\mathbf{R z} \\
{[\boldsymbol{\mu} \mathbf{m}]}\end{array}$ \\
\hline Coated steel ball & 0.023 & 0.240 \\
\hline Strip drawing tool & 0.028 & 0.180 \\
\hline Aluminium sheet & 0.308 & 1.612 \\
\hline
\end{tabular}

The a-C:H coating system was tested afterwards by a strip drawing test to verify the observations of the ball-on-disc tribometer tests under an application nearer test condition. The strip drawing test enables emulating the typical tribological load spectrum of deep drawing and stretch forming processes [12]. In this test, a pressure is applied to a strip of sheet material by an upper and lower tool while the strip is drawn with the defined sliding speed and sliding distance summarized in Table 2. Figure 1 shows the test principle schematically. In order to apply a characteristic load spectrum for sheet metal forming the typical linear load and corresponding high contact pressure at the die radius of a deep drawing tool is reproduced through the cylinder-plane geometry in the strip drawing. The upper tool has a cylindrical surface with a radius $r=258 \mathrm{~mm}$ while the lower tool is flat. The basic measurements of both tools are equal to $40 \mathrm{~mm} \times 40$ $\mathrm{mm}$. Tool material (1.2379) and tested coatings are identical in both tribometer tests and it is equally tested against EN AW 5083 aluminium.

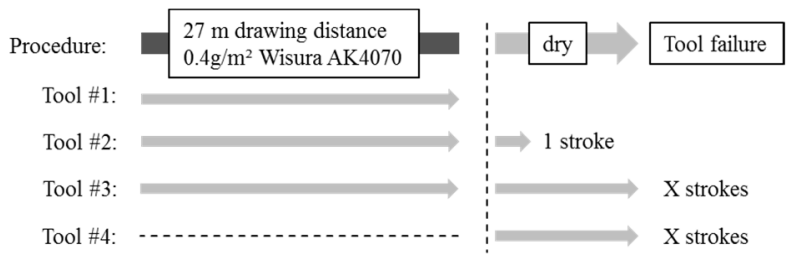

Fig. 1. Test procedure for the strip drawing tests.

The test procedure was chosen according to Fig. 1. After drawing $27 \mathrm{~m}$ under lubricated conditions, tool \#1 was cleaned from lubricant and underwent further investigations regarding the conditions of the coating. Tool \#2 and Tool \#3 were also tested with a lubricated drawing distance of $27 \mathrm{~m}$ and cleaned. While Tool \#2 was tested in one stroke under dry conditions, Tool \#3 was tested until severe wear or strip failure occurred. Tool \#4 were directly tested without lubrication and similar to Tool \#3 continued until severe wear were observable. All tools were coated with the standard a-C:H coating. Every strip-drawing test setup were performed once.

The test parameters were deduced from an industrial forming process of EN AW-5083 aluminium and are summarized in Table 2. The initial contact pressure in the ball-on-disc contact is higher than in the strip drawing test but decreases drastically due to wear of the coating and aluminium sheet. All test substrates were cleaned with ethanol and acetone to ensure a technical pure contact during the tribometer tests.

Table 2. Test parameters of tribological tests.

\begin{tabular}{|l|c|c|}
\hline & $\begin{array}{c}\text { Ball-on-disc } \\
\text { Tribometer }\end{array}$ & $\begin{array}{c}\text { Strip } \\
\text { drawing test }\end{array}$ \\
\hline $\begin{array}{l}\text { Load } \\
\text { [ N ] }\end{array}$ & 4 & 9000 \\
\hline $\begin{array}{l}\text { Initial contact stress } \\
\text { [ MPa ] }\end{array}$ & 480 & 100 \\
\hline $\begin{array}{l}\text { Average velocity } \\
{\left[\text { mms }^{-1} \text { ] }\right.} \\
\text { Humidity } \\
{\left[\begin{array}{c}\text { \% ] } \\
\text { Temperature }\end{array}\right.}\end{array}$ & 50 & 50 \\
{$\left[{ }^{\circ} \mathbf{C}\right.$ ] }
\end{tabular}

\section{Results and discussion}

\subsection{Run-in behaviour in tribometer test}

Oscillating tribometer tests revealed high friction values during the run-in period of the a-C:H coating in an unlubricated sliding contact against aluminium, see Fig. 2. Regarding the friction value, the run-in period can be divided in two phases: a high friction phase (up to 0.9) 
within the first $1.5 \mathrm{~m}$ and a medium friction phase $(\sim 0.4)$ until the end of the run-in period (6 to $7.5 \mathrm{~m}$ ). After the run-in period, the friction value decreased to a steady state level of 0.1 which is a well-known friction value of a-C:H coatings in a dry sliding contact against aluminium [5]. The high friction value during the first period equals the high friction values of uncoated forming tools sliding against aluminium [3]. In case of uncoated forming tools, the high friction values are correlated with the formation of aluminium adhesions on the tool surface, which is the main reason for an early tool failure. However, no aluminium adhesions were detectable in the main contact zone on the a-C:H coated steel ball. Only around the main contact zone did aluminium adhere to the a-C:H coating.

For an investigation of the wear progression during the run-in period further oscillating tribometer tests with incrementally increased sliding distances were conducted, see Fig. 3. Similar to the high friction values already

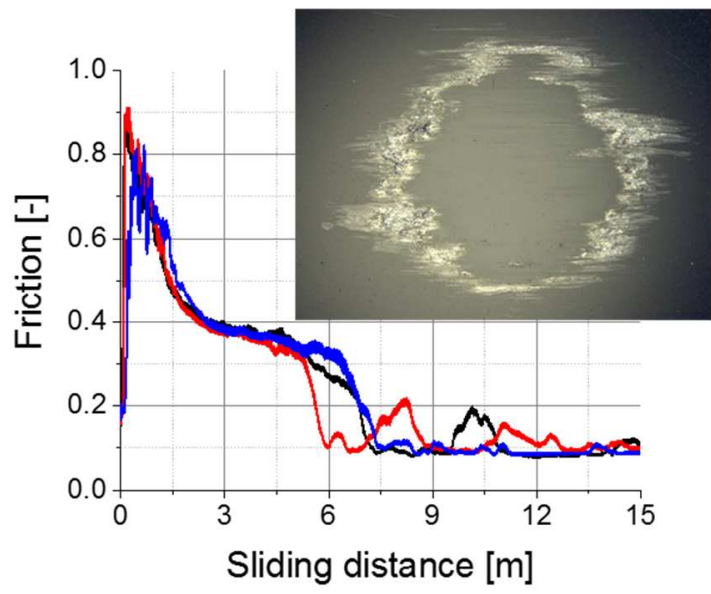

Fig. 2. Oscillating ball-on-disc tribometer tests: Friction development and wear of the a-C:H coated steel ball. indicated, aluminium adhesions were formed on the a$\mathrm{C}: \mathrm{H}$ coated steel ball within a sliding distance of $0.5 \mathrm{~m}$ - covering the complete contact zone, see Fig. 4. During the medium friction phase $(>1.5 \mathrm{~m})$ the amount of aluminium adhesions on the coated steel ball decreased and no further wear of the aluminium sheet was observable with an increasing sliding distance, see Fig. 5. Instead, aluminium accumulated randomly along the aluminium wear track, indicating a retransfer of the aluminium adhesions from the coated steel ball to the wear track. Furthermore, a black transfer layer formed on top of the aluminium accumulations. Reaching the steady state friction level $(>6.5 \mathrm{~m})$, nearly no aluminium adhesions were detectable in the main contact zone comparable to the wear characteristic after $15 \mathrm{~m}$ in Fig. 2.

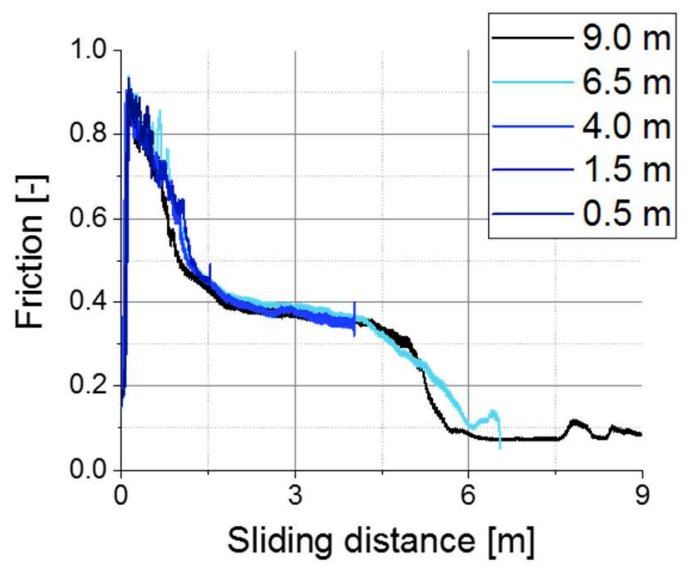

Fig. 3. Friction development of a-C:H during run-in period incrementally increased sliding distances.
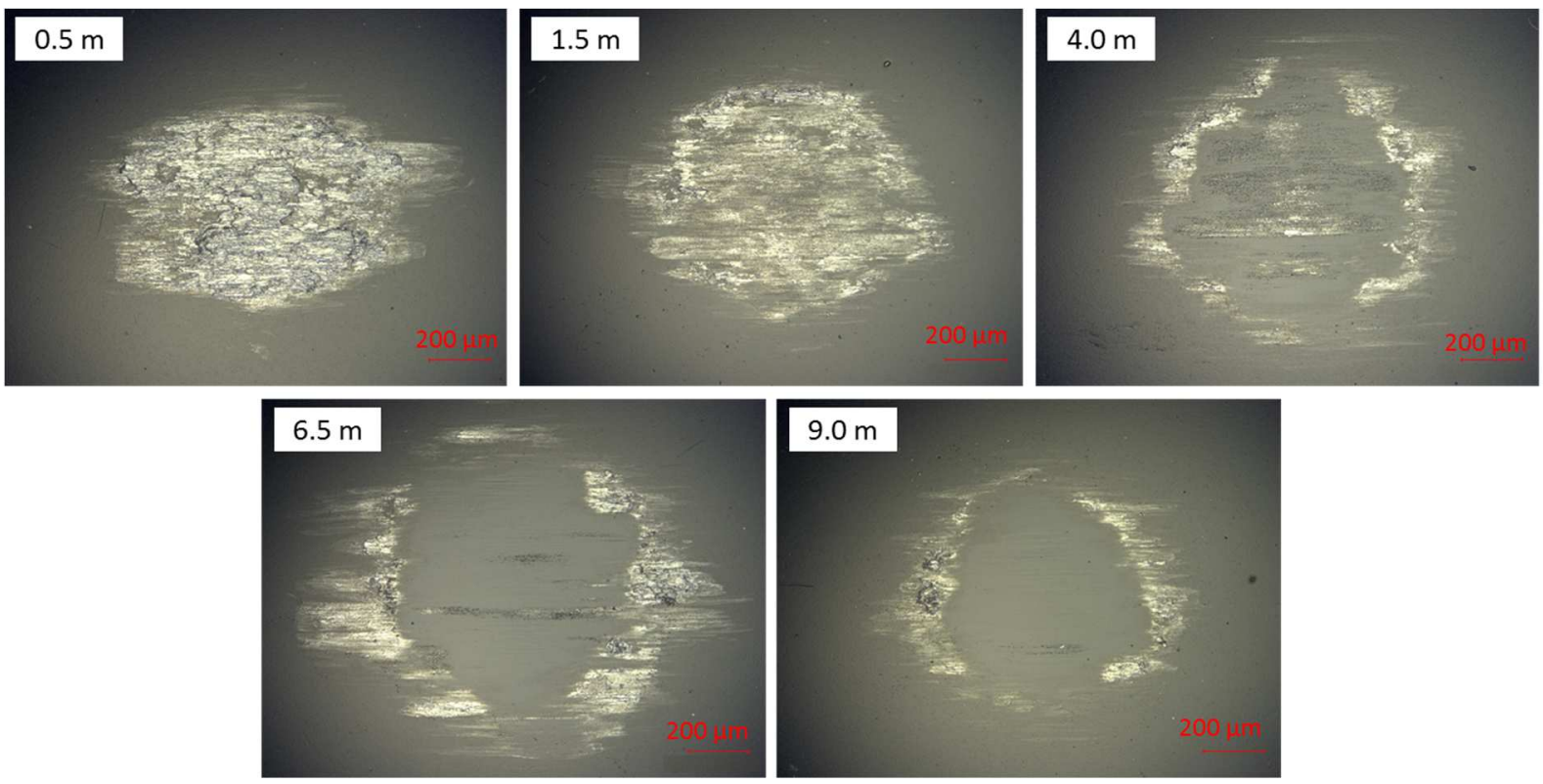

Fig. 4. Microscope pictures of the a-C:H coated steel ball after tribometer tests with incrementally increased sliding distances. 

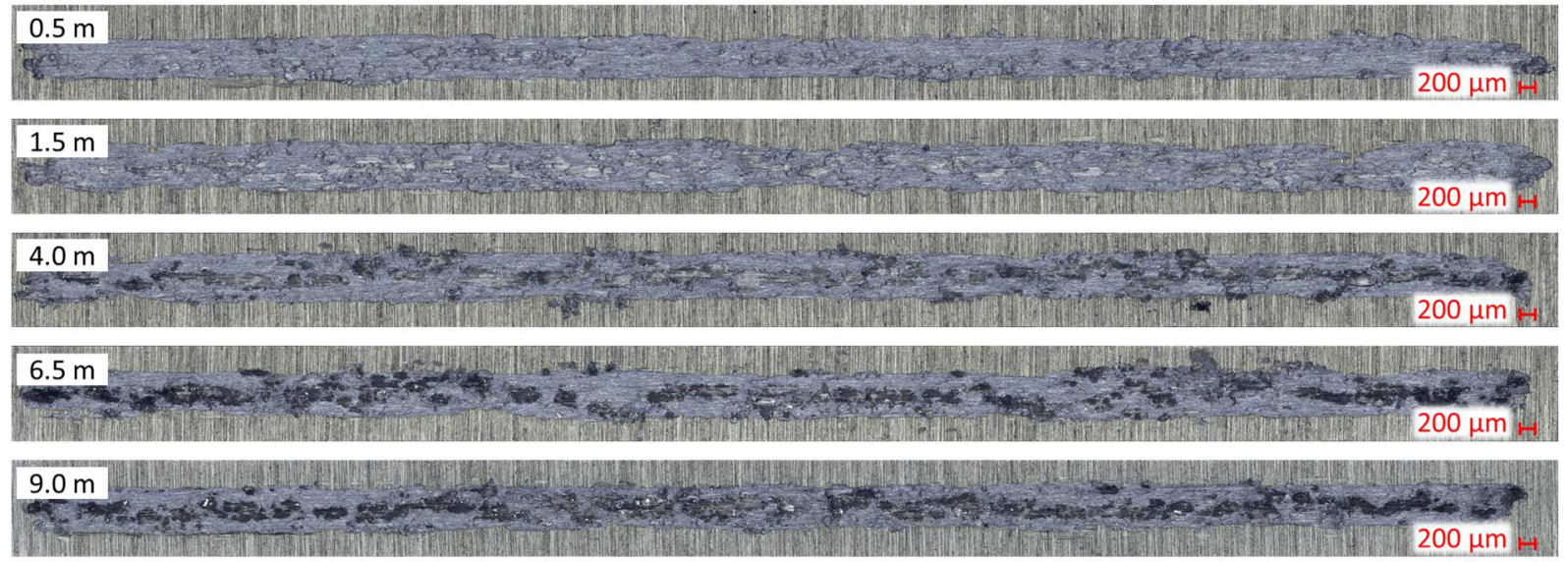

Fig. 5. Microscope pictures of the wear tracks on the aluminium sheets after tribometer tests with incrementally increased sliding distances.
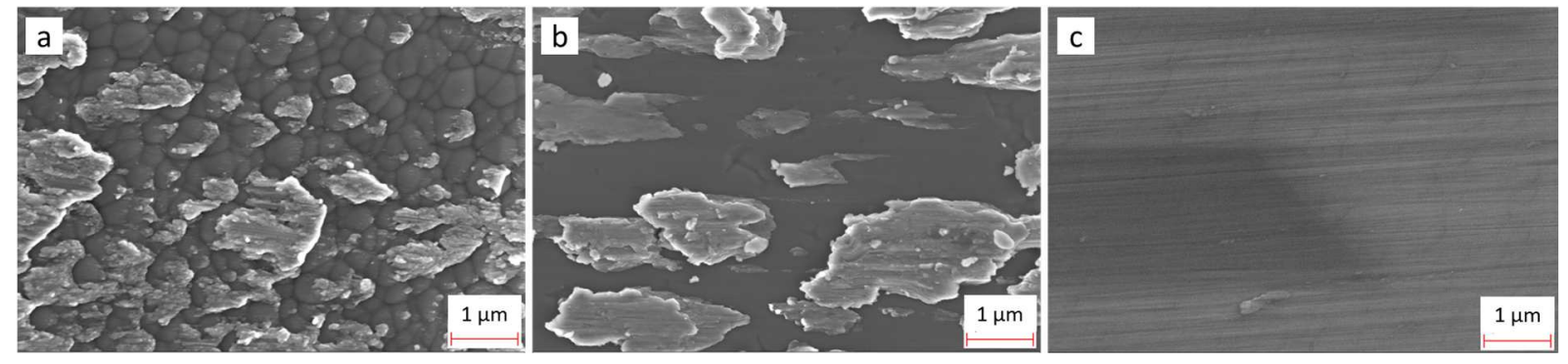

Fig. 6. Scanning electron microscopy (SEM) of the aluminium adhesions formed on the a-C:H coated steel ball after a sliding distance of $0.5 \mathrm{~m} \mathrm{(a),} 4 \mathrm{~m}(\mathrm{~b})$ and $9 \mathrm{~m} \mathrm{(c)}$.

At the beginning of the run-in period, small aluminium adhesions adhered particularly on microscopic domeshaped asperities, see Fig. 6a, which are characteristic for the intrinsic surface structure of the tested a-C:H coating system [13]. These small adhesions may be the initial state for the large laminar structured aluminium adhesions. Meanwhile as the amount of adhesions was reduced, a smoothening of the underlying a-C:H surface structure was observable with an increasing sliding distance, see Fig. $6 \mathrm{~b}$ and Fig. $6 \mathrm{c}$. At the end of the run-in period the main contact area was completely covered with grooves indicating an abrasion of the a-C:H coating. According to this observations, the a-C:H surface structure may promote the formation of aluminium adhesions and thus, may be an important factor for the run-in behaviour of the a-C:H coating in a dry sliding contact against aluminium.

For a more detailed investigation of the observed smoothening progression, the nanometer-scale roughness of all tested steel balls were determined. Therefore, the aluminium adhesions were removed by etching with a sodium hydroxide solution at room temperature. The surface roughness was measured afterwards with an atomic force microscope (AFM) (Veeco Dimension 3100) in tapping mode with a scan size of $10 \times 10 \mu \mathrm{m}$. Focusing on the nanometer-scale a-C:H roughness, the collected data were subsequently processed with a Gaussian filter to subtract the roughness of the steel ball and macroscopic grooves in the a-C:H coating. The measurements revealed a significant reduction of the a-C:H coating roughness during the run-in period, see Fig. 7. The a-C:H asperities were mainly abraded during the medium friction phase dropping from an initially mean height of $15.3 \mathrm{~nm}$ to 1.9 $\mathrm{nm}$ after a sliding distance of $6.5 \mathrm{~m}$.

According to Harris et. al. [14] the intrinsic nanometer-scale roughness of a-C:H coatings is an important tribological property influencing the abrasion of the counter material. In particular, the a-C:H asperities penetrate and consequently abrade the surface of the counter material. In a dry sliding contact against aluminium the a-C:H surface contacts initially the passivated aluminium surface. The passivated surface consists of an inert aluminium oxide layer (A12O3) with a thickness ranging between 2 to $12 \mathrm{~nm}$ [15]. In comparison the reduced peak height of an untested a-C:H coating measured $21.1 \mathrm{~nm}$. Thus, the asperities may penetrate the aluminium oxide layer and contact the pure aluminium matrix. The aluminium matrix is highly reactive and as a consequence may adhere to the a-C:H asperities. However, the a-C:H surface is highly inert due to a passivation by hydrogen and hydroxide [7]. Furthermore, the soft aluminium matrix may be pressed into the surface valleys of the a-C:H coating. Providing a mechanical attachment, the surface valleys may promote the formation of aluminium adhesions. This thesis would explain the observed small aluminium adhesions particularly formed on the a-C:H asperities (Fig. 6a).

As already stated, the aluminium accumulations along the wear track (Fig. 5) indicate a retransfer of the aluminium adhesions from the coated steel ball to the aluminium sheet. Analysis of these accumulations revealed a higher hardness $\mathrm{HUpl}$ of $3.3 \mathrm{GPa}$ compared to the untested aluminium surface with a HUpl of 1.2 GPa. During the transfer processes, these accumulations were 
stressed and frequently exposed to air leading to work hardening and a stronger oxidation. With an increasing amount of aluminium accumulations, the contacting area between the coated steel ball and the aluminium sheet were gradually reduced. In regard to the postulated theory about the promoted adhesion formation by the a-C:H asperities, the adhesion tendency decreased in present of the stronger oxidised aluminium accumulations. Instead, the accumulations abraded the a-C:H coating and the carbonic wear particles formed the observed transfer layer. These wear mechanisms would explain the smoothening effect and lowered friction value during the midsection of the run-in period.

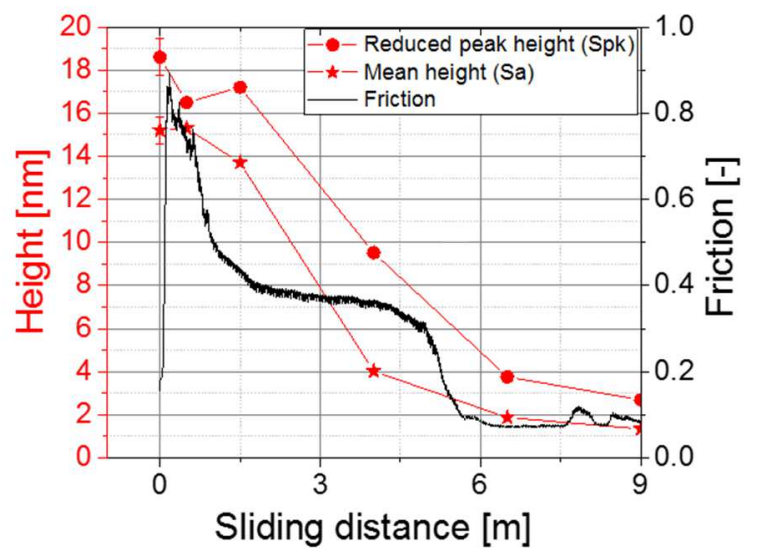

Fig. 7. Smoothening progression of the a-C:H coated steel ball during the run-in period.

Raman spectroscopies were conducted to investigate the atomic structure of the carbonic transfer layer on the aluminium wear track. The measurements were conducted with a laser wavelength of $532 \mathrm{~nm}$ and a laser power of $0.9 \mathrm{~mW}$ revealing a ID/IG-ratio shift from 0.24 for the untested a-C:H coating to 0.81 for the transfer layer [16]. The ratio shift indicates a higher content of the graphitic $\mathrm{sp}^{2}$-configuration in the transfer layer. According to Liu et al. [17], the graphitization leads to a self-lubricating effect lowering the friction value and wear. The delayed graphitization of the a-C:H coating and subsequent formation of a carbonic transfer layer (Fig. 5) are further possible explanations for the high adhesion tendency and high friction values during the run-in period.

In case of real forming processes there is no stationary tribological contact between the coated tool and the aluminium sheet. Thus, the beneficial tribological effect of the carbonic transfer layer should be lowered. To evaluate the impact of the smoothening effect and the graphitization for dry forming processes, further ball-ondisc tribometer tests were conducted with an additional transversal offset added to the oscillating motion to ensure a steady contact with an untested aluminium surface. The elongation of the aluminium sheet in real forming processes enhances significantly the formation of aluminium adhesions [18]. However, there is no elongation of the aluminium sheet in ball-on-disc tribometer tests. To enhance the adhesion tendency, the aluminium sheets were preconditioned by a grinding pad and afterwards cleaned with ethanol and acetone. Consequently, the roughness of the aluminium surface increased $\left(\mathrm{R}_{\mathrm{a}}=0.463 \mu \mathrm{m}\right.$ and $\left.\mathrm{R}_{\mathrm{z}}=4.717 \mu \mathrm{m}\right)$ and the thickness of the native aluminium oxide layer was minimal.

Figure 8a shows the friction development of the $\mathrm{a}-\mathrm{C}: \mathrm{H}$ coating tested under the modified tribometer test conditions. The friction value and adhesion tendency remained for the complete sliding length of $3 \mathrm{~m}$ on a high level similar to the oscillating test with a sliding length of $0.5 \mathrm{~m}$ (Fig. 4). Neither a smoothening of the intrinsic a-C:H roughness nor the formation of a carbonic transfer layer were observable. Aluminium adhesions were steadily formed inhibiting a direct sliding contact at the a-C:H surface. These mechanism may have mitigated a run-in of the a-C:H coating. In an additional test, the $\mathrm{a}-\mathrm{C}: \mathrm{H}$ coating was pre-treated by a prior oscillating test with a sliding length of $9 \mathrm{~m}$, see fig $8 \mathrm{~b}$. The adhesive wear was similar to the a-C:H coating tested for a sliding distance of $9 \mathrm{~m}$ in the stationary test setup (Fig. 5) and no carbonic transfer layer was observable. In addition, the friction coefficient of the pre-treated a-C:H coating continued nearly on the same low level like at the end of the prior oscillating test. Only one sample showed a higher friction value at the beginning which can be explained by a high friction value at the end of the prior oscillating test. As it can be seen in Fig. 2, the friction value sometimes increases for a short distance after the run-in period. This may be caused by a short-term adhesion formation and continued in the following modified tribometer test.

Although the aluminium sheet was changed and the test conditions were non-stationary, the pre-treated a-C:H coating still possessed the optimized tribological properties. According to these results, the run-in behaviour of a-C:H is a unique and temporary effect at the beginning of a dry sliding contact against aluminium. Furthermore, the run-in behaviour and the adhesion tendency of a-C:H are not affected by the formation of a carbonic transfer layer. Still, the graphitization of the a$\mathrm{C}: \mathrm{H}$ boundary surface may influence the tribological properties given that the graphitized state would remain after changing the tribometer test setup. In regard of the fundamental mechanism of the self-lubricating effect, the coating wear would be enhanced due to the graphitization. However, the coating wear did not increase during the modified tribometer tests. Thus, the influence of the graphitization on the run-in behaviour and adhesion tendency of a-C:H coatings is low leaving the smoothening of the intrinsic nanometer-scale a-C:H coating roughness as the most important factor. 

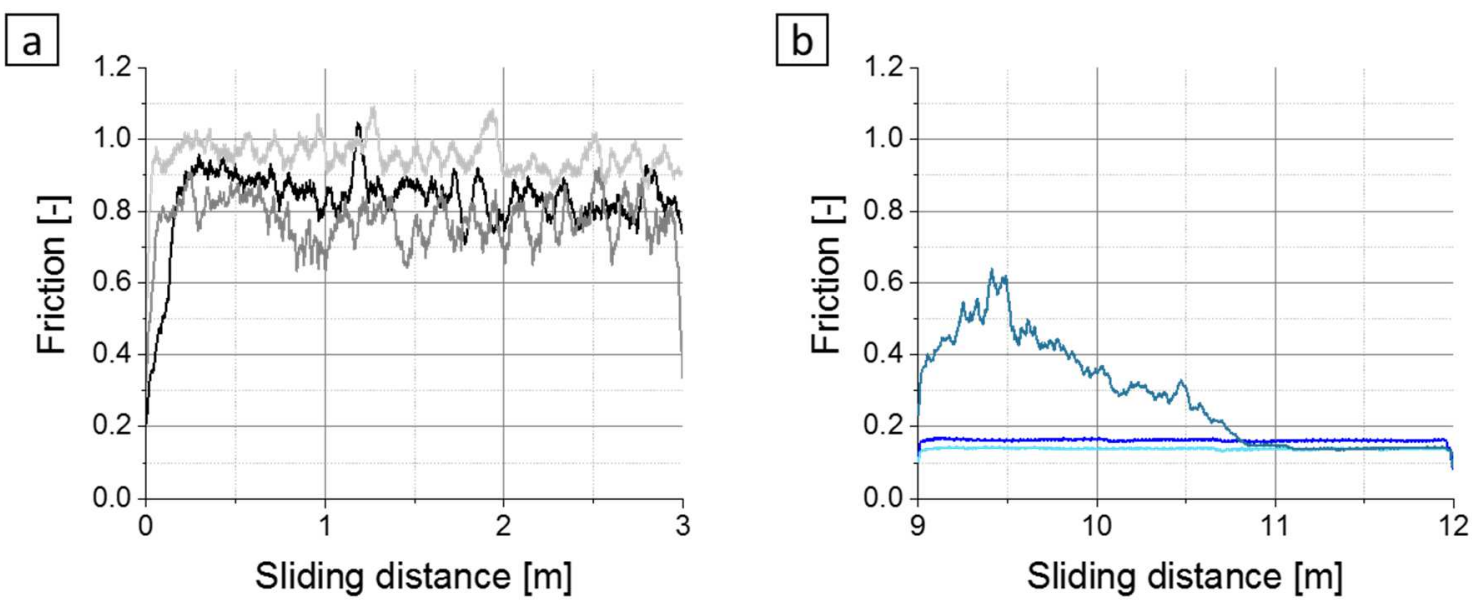

Fig. 8. Friction development of a-C:H (a) and pre-treated a-C:H (b) coated steel balls in modified tribometer tests with forming process nearer test conditions.

As already mentioned, the rapid formation of aluminium adhesions often lead to a premature tool failure in real forming processes. Even the deposition of a state of the art a-C:H coating system shows no significant improvements of the adhesion tendency. According to the tribometer tests, the rapid formation of aluminium adhesions can be explained by a missing smoothening of the intrinsic a-C:H roughness. Consequently, the forming tool fails during the high friction phase of the run-in period when a strong adhesion tendency were observable. To evaluate the impact of the tribological properties during the run-in period on dry forming processes of aluminium further tests were conducted with a strip drawing test, while emulating sheet metal forming conditions more realistically.

\subsection{Run-in behaviour in strip drawing test}

In order to assure lubricant free test conditions for the dry forming processes in Fig. 1, X-ray photoelectron spectroscopies (XPS) of the tool surfaces were performed similar to [19]. Therefore, the tested Tool \#1 were analysed at three different points along the wear track (A, $\mathrm{B}$ and $\mathrm{C}$ ) and compared with an untested a-C:H coating. According to these tests, no significant residues of lubricant additives were detectable after cleaning.

The pre-treated Tools \#1, \#2 and \#3 (before stroke 14) showed significantly less wear than the corresponding Tool \#4 without a prior run-in. This can be observed in the microscopic images taken of the worn tool surface shown in Fig. 9. The darker colour on the Tool \#1 marks the contacting area with the aluminium sheets. Nearly no aluminium adhesions were formed and some isolated deep grooves in sliding direction were observable. Partly the a-C:H coating showed cracks and signs of delamination along the groove edges. These effects may be caused by a contamination of the aluminium surface with hard particles leading to a coating overload. Tool \#2 additionally was drawn one stroke under dry conditions. In comparison to Tool \#1, Tool \#2 slightly more aluminium adhesions adhered to the surface but the amount of adhesions was significantly lower compared with an untreated a-C:H coated tool. Here, the dry sliding contact against aluminium leads to a severe adhesive wear already after one stroke (100 mm sliding length), see [3]. After 75 strokes, the wear appearance did not differ, presented by Tool \#4. In order to find the maximum of possible sliding length without severe adhesive wear the dry sliding length with pre-treated a-C:H coating was extended. After 89 strokes under dry conditions the strip drawing test was interrupted due to a severe adhesive wear which already started to form after 14 strokes. In comparison to the untreated tools, small areas between the adhesions still showed no adhesions. Furthermore, no transfer layer was observable in all tests. Thus, the transfer layer did not affect the tribological performance.

For an investigation of the wear mechanisms during the dry strip drawing tests, the friction values were calculated based on the normal load and the pulling force. Figure 10 shows the average friction development for Tool \#3 during the first 24 strokes under dry conditions. The dashed line represents the friction value of the untreated Tool \#4 $(\mu \sim 0.37)$. The dash-dotted line represents friction value of Tool \#1 tested under lubrication. The squares show the development of the average maximum peak roughness $\mathrm{RZ}$ of the sheet metal for each stroke.

For the first 14 strokes under dry conditions, the friction value laid steadily around 0.1 - slightly above the lubricated case. Beside of small grooves on the sheet metal caused by minor adhesions on the tool surface, there was almost no wear observable, especially compared to the results of Tool \#4. After the first groove in the right side had developed, this groove dominated the $\mathrm{R}_{\mathrm{Z}}$ value of the sheet metal. Thus, the $\mathrm{R}_{\mathrm{z}}$ value did not change much during stroke 4 to 16 corresponding with the observed wear and friction values. After stroke 14 the friction value and $\mathrm{R}_{\mathrm{z}}$ values raised indicating a formation of aluminium adhesions on the tool surface. After 21 strokes, the friction value and the wear of the aluminium sheet was similar to the untreated Tool \#4. In respect to real forming processes, these tribological properties may lead to a tool failure. 

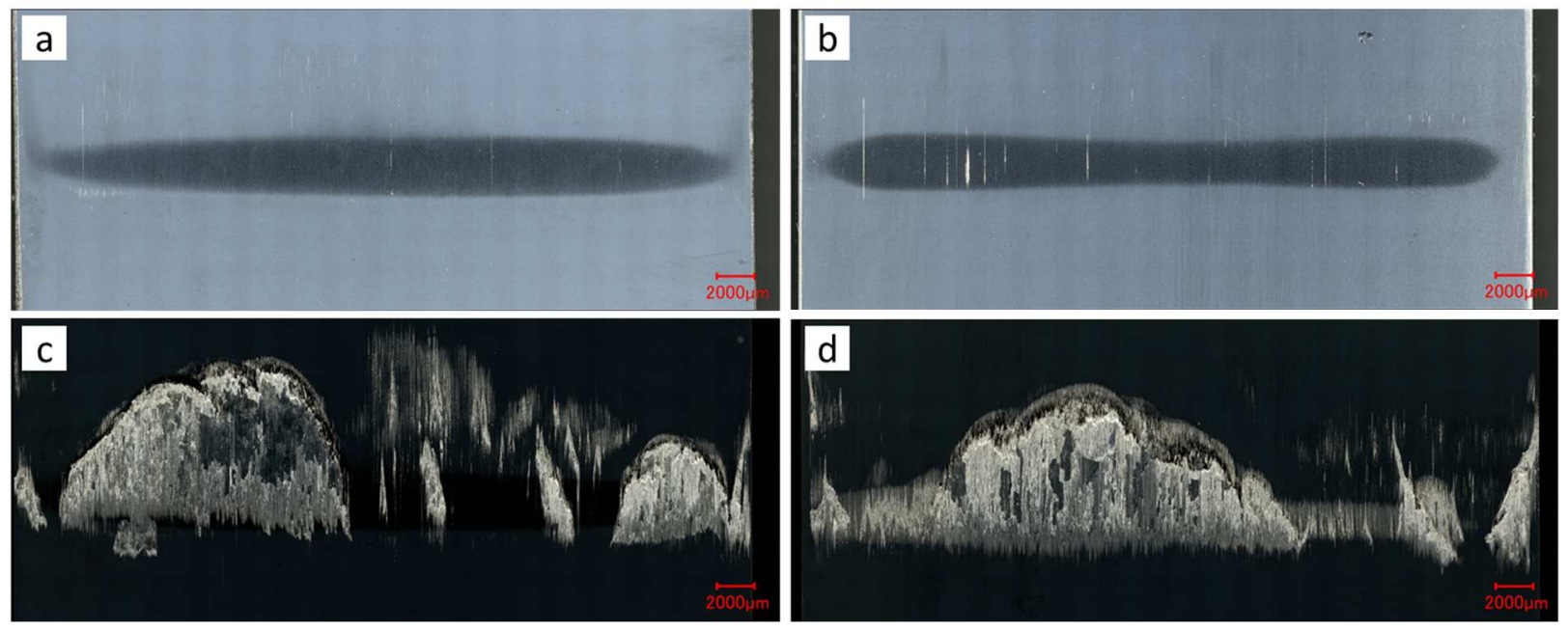

Fig. 9. Microscopic images of the worn tool surfaces. a: Tool \#1, b: Tool \#2, c: Tool \#3, d: Tool \#4.

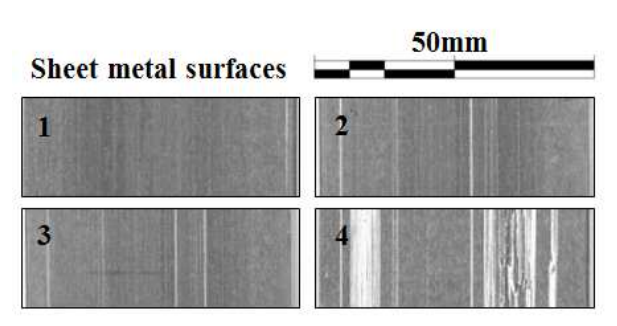

$$
\begin{aligned}
& -\mu, \text { Tool \#3, dry }[] \\
& -\cdots-\mu \_ \text {mean, Tool } \# 1, \text { lubricated }[] \\
& ---\mu \_ \text {mean, Tool } \# 4, \text { dry }[] \\
& \square \quad \text { Rz, sheet metal }[\mu \mathrm{m}]
\end{aligned}
$$

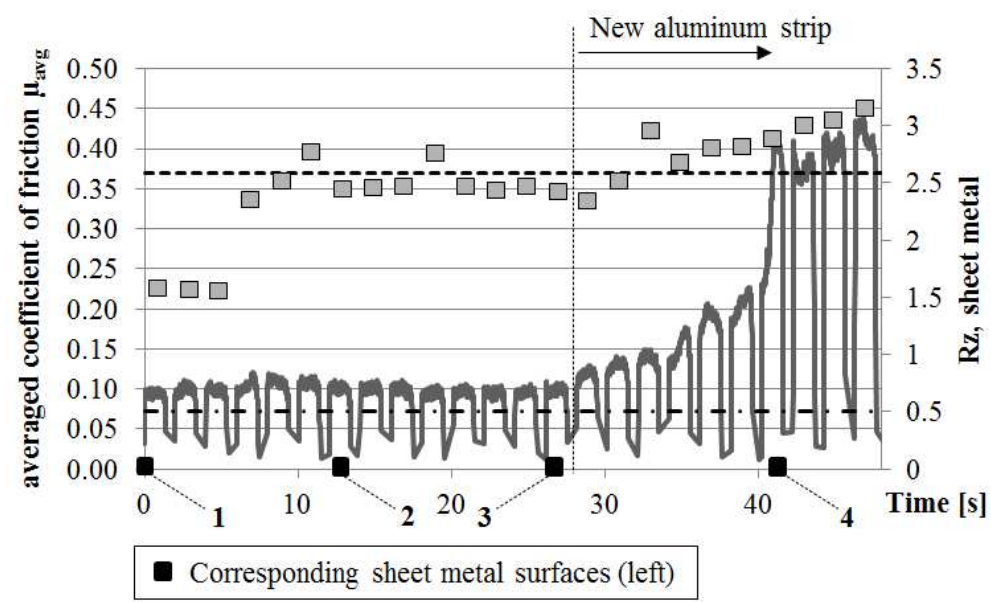

Fig. 10. Development of the coefficient of friction for Tool \#3 including the lubricated reference case of Tool \#1 and \#5.

Analog to the oscillating tribometer tests, the nanometerscale roughness of the tested strip drawing tools were determined by AFM measurements, see Fig. 11. All tools showed a smoothened surface roughness. The mean height of Tool \#1, \#2 and \#3 was equal to the surface roughness of the tested steel balls at the beginning of the medium friction phase (Fig. 7) and most of the intrinsic nanometer-scale a-C:H surface structure was intact, see Fig. 12. The eased smoothening effect may be caused by the lower contact pressure of the strip drawing test and the lubricant. Furthermore, numerous line-shaped areas with unworn a-C:H surfaces were observable in the contact zone. Each line started at coating defects, which were caused by contaminations during the deposition process. These defects possibly deformed the aluminium sheet and lowered the contact stress in the following contact zone. Thus, the smoothening process was partially inhibited. The surface roughness of Tool \#4 was also reduced. Like in the modified tribometer tests, the aluminium adhesions may have mitigated the abrasive wear of the a-C:H coating.

The initial tribological properties of the preconditioned Tool \#2 and \#3 differed from the untreated Tool \#4 and [3]. The reduced initial surface roughness, represented by Tool \#1, lowered the adhesion tendency and friction value significantly for a sliding distance of $1.4 \mathrm{~m}$ matching the performance of the pre-treated a-C:H coating in the modified tribometer test. The abrupt aggravation of the tribological performance of Tool \#3 can possibly be explained by the setup of the strip drawing test. After 14 strokes, the aluminium test strip had to be changed for a new strip (Fig.10). Therefore, the press was opened and the next strip was fed to the gripping device. By changing the strip, the line-shaped unworn a-C:H surface areas on Tool \#3 contacted the aluminium sheet. These unworn a-C:H areas still possessed the high adhesion tendency and may have promoted the adhesion formation. Regarding Tool \#2, this effect may already have caused the formation of small adhesions during the first aluminium strip but the amount of adhesions were maybe too little to enhance the adhesion formation. Continuing with the second strip, a certain amount of adhesions was reached promoting the adhesion formation progressively. 


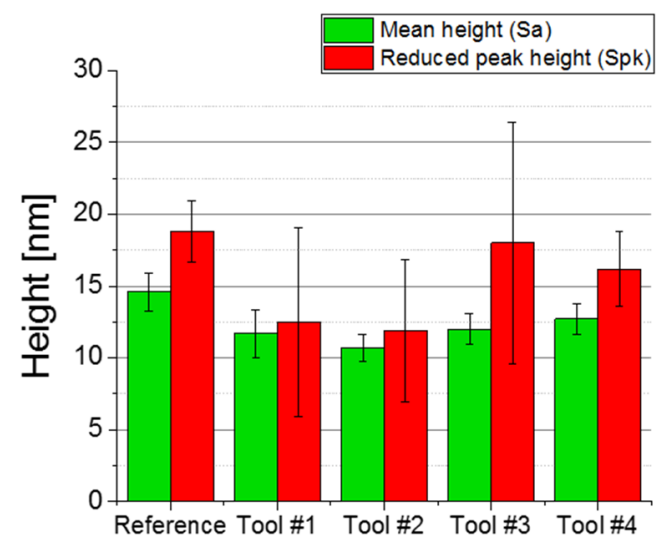

Fig. 11. Nanometer-scale roughness of the tested strip drawing tools.

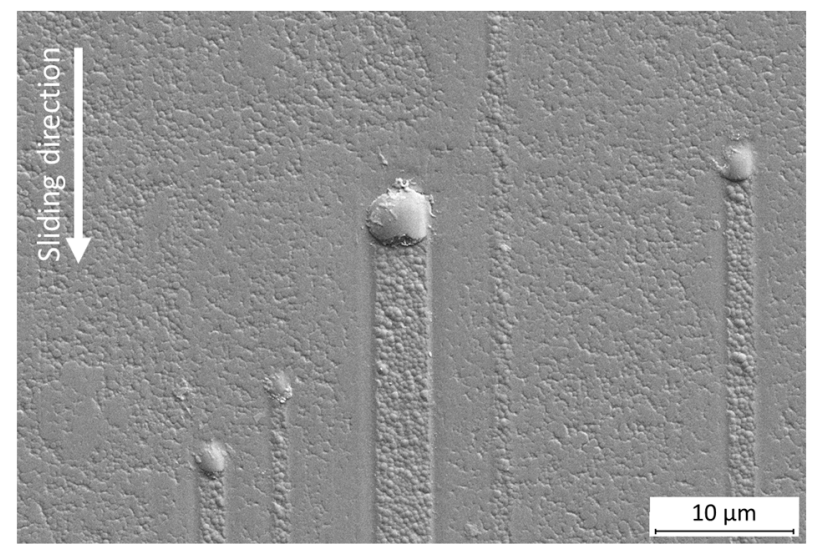

Fig. 12. SEM image of Tool $\# 1$ - coating defects inhibited the smoothening process during lubricated strip drawing tests (sliding direction: top to down).

\section{Conclusion}

In this paper the run-in behaviour of a-C:H used as tool coating for dry forming processes of aluminium sheets was investigated and as a possible reason for a premature tool failure evaluated. Oscillating ball-on-disc tribometer tests revealed a high friction value and strong adhesion tendency during the run-in period. The aluminium adhesions adhered particularly on the dome-shaped asperities of the intrinsic nanometer-scale a-C:H surface roughness. During the run-in period, the intrinsic coating roughness was completely abraded leading to a significantly smoother surface and a graphitic transfer layer connected with a very low adhesion tendency and a low friction value after the run-in period. In subsequent non-stationary tribometer tests, these properties remained on the same level and no transfer layer was observable. Thus, the run-in period of a-C:H is a unique and temporary effect at the beginning of a dry sliding contact against aluminium. Furthermore, the intrinsic nanometerscale a-C:H surface roughness may be a crucial factor determining the adhesion tendency and friction value during the run-in period. These interdependencies can be explained by a grooving of the aluminium oxide layer caused by the coating asperities. The subsequent contact with pure aluminium may promote a chemical attachment whereas the surface valleys may promote a mechanical attachment for the deformed aluminium. Although no transfer layer was observable, a tribological induced graphitization of the a-C:H boundary surface connected with self-lubricating properties cannot be excluded as an additional factor. Though, no wear of the coating was observable after the run-in period, the influence of a tribological induced graphitization may be little.

These wear mechanisms were also verified by strip drawing tests, which emulate sheet metal forming conditions more realistically. Based on a tool pretreatment, the intrinsic nanometer-scale a- $\mathrm{C}: \mathrm{H}$ roughness were smoothened leading to a significantly reduced friction value and adhesion tendency. However, the pretreatment was insufficient to enable dry forming of aluminium. Due to coating defects, small areas of the intrinsic a-C:H roughness were still intact promoting a delayed adhesion formation.

According to these results, the intrinsic nanometerscale a-C:H surface roughness promotes the rapid adhesion formation in dry sliding contacts against aluminium and thus determines the tribological performance of a-C:H tool coatings in dry sheet metal forming of aluminium. The rapid formation of aluminium adhesion mitigates the smoothening due to abrasion. In consequence, the premature tool failure occurs before falling under a certain critical a-C:H coating roughness. Thus, dry metal sheet forming of aluminium may be enabled by the usage of very smooth a-C:H tool coatings.

In order to validate this hypothesis, further tests with smoother a-C:H coatings are necessary. By extending the tool pre-treatment method, the obtainable a-C:H surface roughness can be improved but is limited to the amount of surface defects. A more appropriate approach is a direct deposition of smooth a-C:H coatings based on an optimization of the deposition parameters. Alternatively, the intrinsic a-C:H roughness can possibly be lowered by plasma etching in a post-treatment process.

\section{Acknowledgements}

The authors would like to thank the German Research Foundation (DFG) for funding of the project "Functionalising of a-C:H tool coatings and homogenization of the aluminium passive layer for the dry forming of aluminum" received within the priority program SPP 1676 "Dry metal forming - sustainable production through dry processing in metal forming".

\section{References}

1. P. Groche, F. Resch, Mat.-wiss. u. Werkstofftech 46, 813-828 (2015)

2. K. Bewilogua, D. Hofmann, Surface \& Coatings Technology 242, 214-225 (2014)

3. T. Abraham, G. Bräuer, F. Kretz, P. Groche, Dry Met. Forming OAJ FMT 2, 18-24 (2016)

4. A. Wank, G. Reisel, B. Wielage, Surface \& Coatings Technology 201, 822-827 (2006) 
5. H. Ronkainen, S. Varjus, K. Holmberg, Wear 222, 120-128 (1998)

6. H. Ronkainen, J. Likonen, J. Koskinen, S. Varjus, Surface \& Coatings Technolgy 79, 87-94 (1996)

7. A. Erdemir, Surface \& Coatings Technology 146147, 292-297 (2001)

8. S.Y. Luo, J.K. Kuo, T.J. Tsai, C.W. Dai, Wear 249, 800-807 (2001)

9. J. Goldsmith, E. Sutter, J.J. Moore, B. Mishra, M. Crowder, Surface \& Coatings Technology 200, 23862390 (2005)

10. K. Taube, Surface \& Coatings Technology 98, 976984 (1998)

11. T. Michler, C. Siebert, Surface \& Coatings Technology 163-164, 546-551 (2003)

12. P. Groche, J. Filzek, G. Nitzsche, Annals of the German Academic Society for Production Engineering XI/1 11, 55-60 (2004)

13. M. Weber, K. Bewilogua, H. Thomsen, R. Wittorf, Surface \& Coatings Technology 201, 1576-1582 (2006)

14. S.J. Harris, A.M. Weiner, C.H. Olk, M. Grischke, Wear 219, 94-104 (1998)

15. K. Wefers, Aluminium 57, 722 (1981)

16. A.C. Ferrari, J. Robertson, Physical Review B 61, 20 (2000)

17. Y. Liu, A. Erdemir, E.I. Meletis, Surface \& Coatings Technology 86-87, 564-568 (1996)

18. N. Bay, AWS Annual Meeting 63, 137-142 (1982)

19. A. Almohallami, M. Arghavani, F. Böhmermann, H. Freiße, M. Herrmann, S.A. Mousavi, S. Schöler, P. Scholz, J. Tenner, M. Teller, G. Umlauf, D. Wulff, D. Yilkiran, H.J. Maier, Dry Met. Forming OAJ FMT 3, 90-94 (2017) 